\title{
Conducto con exudado persistente: Causas y manejo clínico
}

\author{
Weeping canal: Causes and clinical management \\ Ducto com exsudato persistente: Causas e manejo clínico
}

Recibido: 22/07/2021 | Revisado: 25/07/2021 | Acepto: 25/07/2021 | Publicado: 02/08/2021

\author{
José Luis Álvarez Vásquez \\ ORCID: https://orcid.org/0000-0003-0381-2402 \\ Universidad de Cuenca, Ecuador \\ E-mail: jose.alvarezv@ucuenca.edu.ec \\ María Cristina Guazhima Fernández \\ ORCID: https://orcid.org/0000-0001-9710-1497 \\ Universidad de Cuenca, Ecuador \\ E-mail: cristina.guazhima96@ucuenca.edu.ec \\ Natasha Carolina Durán Ortiz \\ ORCID: https://orcid.org/0000-0002-7678-680X \\ Universidad de Cuenca, Ecuador \\ E-mail: natasha.duran@ucuenca.edu.ec
}

\begin{abstract}
Resumen
Objetivo: El objetivo de esta revisión integrativa de la literatura fue abordar las causas y manejo clínico del conducto con exudado persistente o "conducto llorón", que es una entidad clínica caracterizada por la presencia intraconducto de exudado inflamatorio persistente, pese al tratamiento de conducto en ejecución, incluso después de varias citas. Metodología: Se realizó un levantamiento bibliográfico en las bases de datos Pubmed, Scopus y EBSCO, en idioma inglés y sin restricción de fecha de publicación. Los términos de búsqueda empleados en combinación fueron: conducto húmedo, conducto llorón, exudado persistente, exudación intraconducto persistente y, tratamiento de conducto radicular. Resultados y conclusión: Se concluye que la causa principalmente descrita es la microbiana, en forma de biofilm bacteriano o infección recalcitrante. Respecto a su manejo clínico, existen disponibles una serie de abordajes, como las puntas de papel, la medicación intraconducto y sistemas de aspiración como el EndoVac, en tanto que el empleo de la terapia con láser Nd:YAG requiere de más estudios que respalden su uso clínico.
\end{abstract}

Palabras clave: Exudado persistente; Tratamiento de conducto radicular; Endodoncia.

\begin{abstract}
Objective: The aim of this integrative literature review was to address the causes and clinical management of the canal with persistent exudate or "weeping canal", which is a clinical entity characterized by the presence of persistent inflammatory exudate in the canal, despite ongoing root canal treatment even after multiple appointments. Methodology: A bibliographic survey was carried out in the Pubmed, Scopus and EBSCO databases, in English and without restriction of publication date. The search terms used in combination were: wet canal, weeping canal, persistent exudate, persistent intracanal exudation, and root canal treatment. Results and conclusion: It is concluded that the main cause described is microbial, in the form of bacterial biofilm or recalcitrant infection. Regarding its clinical management, a series of approaches are available, such as paper points, intracanal dressing, and aspiration systems such as Endovac, while the use of Nd:YAG laser requires more studies to support its clinical use.

Keywords: Persistent exudate; Root canal therapy; Endodontics.

\section{Resumo}

Objetivo: O objetivo desta revisão integrativa da literatura foi abordar as causas e o manejo clínico do ducto com exsudato persistente ou "ducto lacrimejante", que é uma entidade clínica caracterizada pela presença de exsudato inflamatório persistente no ducto, apesar do ducto tratamento em execução, mesmo após várias consultas. Metodologia: Foi realizado levantamento bibliográfico nas bases de dados Pubmed, Scopus e EBSCO, em inglês e sem restrição de data de publicação. Os termos de pesquisa usados em combinação foram: canal radicular úmido, canal de drenagem, exsudato persistente, exsudato intracanal persistente e tratamento de canal radicular. Resultados e conclusão: Conclui-se que a principal causa descrita é microbiana, na forma de biofilme bacteriano ou infecção recalcitrante. Em relação ao seu manejo clínico, uma série de abordagens estão disponíveis, como pontas de papel, medicação intracanal e sistemas de aspiração como o EndoVac, enquanto o uso da terapia a laser Nd: YAG requer mais estudos para embasar seu uso clínico.
\end{abstract}

Palavras-chave: Exsudato persistente; Tratamento do canal radicular; Endodontia. 
Research, Society and Development, v. 10, n. 9, e52910918558, 2021

(CC BY 4.0) | ISSN 2525-3409 | DOI: http://dx.doi.org/10.33448/rsd-v10i9.18558

\section{Introducción}

El conducto con exudado persistente, denominado también "conducto llorón” (Gutmann \& Lovdahl, 2014; Tronstad, 2003; Gulabivala \& Ng, 2014; Durack \& Brady, 2019; Walton \& Keiser, 2009) es una condición clínica caracterizada por la filtración continua de exudado inflamatorio seroso persistente en el conducto radicular, pese al tratamiento de conducto en ejecución, incluso después de varias citas. Puede ser causado por una infección bacteriana, pero también puede asociarse a un foramen apical amplio, ya sea este natural o sobreinstrumentado (causa mecánica (Alshahrani et al., 2016)), en comunicación con un quiste en bolsa (Siqueira \& Rôças, 2016; Riccuci et al., 2016). Otra causa que se ha citado en la literatura es la química, en relación al empleo de antisépticos de tipo aldehídos y fenólicos durante la terapia endodóntica (Tronstad, 2003).

La presencia física de esta exudación, que es refractaria al tratamiento endodóntico ortógrado, crea un ambiente húmedo, que impediría un correcto sellado al momento de la obturación e indica que el tratamiento no está siendo efectivo en controlar la causa de la inflamación perirradicular (Siqueira et al., 2015). Existen disponibles algunos abordajes para el manejo clínico del exudado persistente, como el uso de las puntas de papel, la medicación intraconducto, sistemas de aspiración como el Endovac y terapia con láser Nd:YAG .

En este sentido, la presente revisión integrativa de la literatura tuvo como objetivo abordar las causas y manejo clínico del conducto con exudado persistente, que es una entidad que en ocasiones se presenta durante la terapia endodóntica y se caracteriza por la presencia intraconducto de exudado inflamatorio persistente, sobre el cual el profesional odontólogo puede tener dudas precisamente acerca de sus causas y manejo clínico.

\section{Metodología}

El presente artículo tiene un enfoque metodológico cualitativo. Siguiendo las recomendaciones de algunos autores (Estrela, 2018; Pereira et al., 2018; Koche, 2011) se realizó una revisión integrativa de la literatura mediante una búsqueda electrónica de artículos científicos y libros que contengan la información requerida, empleando las bases de datos PubMed, Scopus y EBSCO, en idioma inglés y sin restricción de fecha de publicación. Los términos de búsqueda empleados en combinación fueron "wet canal", "weeping canal", "persistent exudate", "persistent intracanal exudation" y "root canal treatment". Los términos relacionados fueron combinados usando los operadores "OR y "AND”. Después de la selección inicial de títulos y resúmenes, se removieron duplicados y se seleccionaron únicamente artículos y libros de mayor relevancia y pertinencia para el tema en revisión; los artículos fueron categorizados de acuerdo a las secciones de la presente revisión.

\section{Resultados y discusión}

Se estudió y analizó el texto completo de 17 artículos seleccionados, así como la información pertinente de 8 libros escogidos, con el fin de proveer a los lectores la más reciente y relevante información sobre el conducto con exudado persistente. Los contenidos a tratar serán abordados en dos secciones: causas y el manejo clínico de esta entidad.

\section{Etiología}

La literatura ha evidenciado algunos agentes causales del conducto con exudado persistente, los cuales serán abordados a continuación, pudiendo llegar a presentarse una combinación de los mismos, dependiendo del caso clínico en cuestión. 


\section{Causa bacteriana o infección recalcitrante}

La presencia de exudado inflamatorio persistente en el conducto, después de realizada la preparación quimiomecánica, es altamente sugestiva de la presencia de una infección recalcitrante, especialmente si este exudado es purulento (Siqueira \& Rôças, 2016). Si existen bacterias residuales en la parte más apical del conducto radicular o en sus ramificaciones, estas tienen acceso no restringido a nutrientes, en forma de proteínas y glicoproteínas presentes en los fluidos tisulares y exudados (Siqueira \& Rôças, 2020).

Ricucci et al., 2016 sostienen que la causa de un exudado persistente es posiblemente una infección constante, como lo evidencian en el reporte de dos casos clínicos, que contribuyen significativamente al entendimiento de la etiología. En estos casos reportados, que involucraban dientes con lesiones periapicales, incluso luego de varias citas de terapia endodóntica y medicación intraconducto, los dientes tenían exudación persistente intraconducto. El análisis histológico e histobacteriológico permitieron determinar la causa, esto es, la presencia de biopelícula apical intrarradicular, en continuidad con una gruesa biopelícula extrarradicular mineralizada (cálculo extrarradicular).

Por otro lado, hay que considerar que la mayoría de casos de biofilm extrarradicular están asociados con tractos sinusales, casos en lo que podría ocurrir una egresión de bacterias patogénicas desde el conducto hacia los tejidos perirradiculares. Además, la presencia bacteriana a nivel extrarradicular podría verse incrementada debido a una sobreinstrumentación (Siqueira \& Rôças, 2013).

En un caso reportado en una serie de casos (Santos Soares et al., 2016) que involucraba una diente \# 22 con una gran lesión periapical que semejaba un quiste, hubo fracaso endodóntico ya que se presentó una lesión refractaria, que seguía drenando durante 4 meses continuamente exudado inflamatorio periapical, pese a los esfuerzos por controlar la infección mediante una mezcla de medicación intraconducto de hidróxido de calcio/clorhexidina. El diente finalmente requirió cirugía periapical, luego de la cual el examen microscópico reveló un exuberante biofilm extrarradicular, adherido al ápice radicular seccionado, así como una estructura quística conteniendo cristales de colesterol (Santos Soares et al., 2016). Las posibles causas de fracaso del tratamiento podrían incluir una resistente infección intraconducto (Signoretti et al., 2013; Sousa et al., 2014; Zhanget al., 2015) y/o una infección extrarradicular (Bhaskar, 1972), en forma de biofilms bacterianos (Natkin et al., 1984; Lomçali et al., 1996; Ferreira et al., 2004).

En otro caso reportado (Kahler, 2015) que incluía un diente \#12 con una gran lesión periapical que semejaba un quiste, al realizar la apertura cavitaria hubo abundante drenaje con exudado purulento. Luego de una hora de finalizada la preparación quimiomecánica, el exudado del conducto persistía, por lo que se colocó hidróxido de calcio como medicación intraconducto. Debido a que en citas subsecuentes el diente presentaba exudado purulento persistente, de color verdoso/amarillo, fueron requeridas 3 citas más con hidróxido de calcio, hasta conseguir un conducto seco, previo a la obturación del mismo. Aunque en este estudio no se efectuó un cultivo, se plantea desde una perspectiva bacteriológica que la presencia de exudado purulento se ha asociado con bacterias negras pigmentadas (Sundqvist et al., 1989; Baumgartner et al., 1999) como Porphyromonas, Peptostreptococcus y Fusobacterium spp (Gomes et al., 2004), Peptostreptococcus, Prevotella, Propionibacterium spp. y, bacterias facultativas (Gomes et al., 1994). Finalmente, en este caso reportado se consiguió éxito en la reparación ósea de la gran lesión periapical, la cual incluso involucraba a un implante adyacente a la lesión (Kahler, 2015).

\section{Causa mecánica}

Tras la preparación del conducto radicular, eventualmente puede existir la presencia de exudado inflamatorio persistente, de manera que la obturación debe posponerse, caso contrario la incorporación de exudado al momento de la obturación 
comprometería el sellado, proporcionándose así a los microorganismos de un espacio para multiplicarse, así como de una fuente de nutrientes (Durak \& Brady, 2019).

Una de las causas más comunes para que existan conductos húmedos o sangrantes, es la transportación del foramen apical, que puede dar como resultado una inflamación persistente crónica y el fracaso de la terapia endodóntica (Alshahrani et al., 2016). La excesiva instrumentación ocasionada por el transporte apical, permite que los fluidos tisulares retroceden hacia al conducto, por el daño en los tejidos periapicales. Con un foramen apical que se ha agrandado de forma repentina, el fluido puede ingresar al conducto y hacer difícil la obturación y el sellado del mismo. Además, si los tejidos periapicales han sido inoculados con bacterias provenientes del conducto radicular, el "conducto llorón" podría deberse a una destrucción localizada del tejido, producida por la invasión bacteriana (Gutmann \& Lovdahl, 2014).

\section{Causa química}

Antisépticos de tipo aldehídos y fenólicos, utilizados en el conducto radicular, afectan sin distinción tanto a bacterias como células del huésped. En consecuencia, pueden causar una inflamación inducida químicamente en los tejidos periapicales, con exudación persistente hacia el conducto radicular. Al momento de diagnosticar la exudación periapical inducida químicamente, se puede llegar a confundir de forma errónea con una periodontitis apical infecciosa, y para eliminar las supuestas bacterias, el clínico podría pensar en el empleo de más citas con estos antisépticos, agravando la afectación tisular (Tronstad, 2003).

\section{Manejo clínico}

Existen disponibles en la literatura algunas opciones para el manejo clínico del conducto con exudado persistente, las cuales se describen a continuación. Estas opciones pueden llegar a combinarse, según sea el caso clínico del que se trate.

\section{Puntas de papel}

Es inherente que una opción intraoperatoria de primera mano constituye el empleo de las puntas de papel, las cuales poseen celulosa vegetal en su composición, misma que es un polímero altamente hidrofílico y por ende les confiere las propiedades de absorción que aquellas poseen (Siqueira, 2001; Brown, 2017). No obstante, hay que tener precaución con el empleo de las puntas de papel para no tocar los tejidos periapicales con ellas, ya que la celulosa no puede ser degradada por las células del sistema inmune (Siqueira, 2001) y podría entonces provocar reacciones a cuerpo extraño y ser posible causa de persistencia de lesiones perirradiculares (Siqueira, 2001; Brown, 2017; Koppang, et al., 1988).

En razón de lo antes indicado, se ha referido que deberían realizarse más investigaciones para fabricar puntas de papel que sean biocompatibles (Brown, 2017). En este sentido, una investigación ha evidenciado el potencial uso de puntas de papel fabricadas con celulosa bacteriana durante el tratamiento endodóntico, ya que éstas puntas demostraron una biocompatibilidad más alta, una tasa de absorción 10 veces mayor que las puntas de papel convencionales a base de celulosa vegetal, así como mantuvieron una alta fuerza tensil, incluso cuando estaban ya húmedas (Yoshino et al., 2013).

\section{Medicación intraconducto}

Según Weine, 2004, un medicamento intraconducto es un agente antimicrobiano que se coloca dentro del conducto radicular, entre citas de un tratamiento endodóntico, en un intento de destruir los microorganismos residuales y prevenir la reinfección. La presencia de un exudado persistente en el conducto después de la preparación quimiomecánica, es indicativo de una 
infección recalcitrante, especialmente si el exudado es purulento. La medicación intraconducto se utiliza en estos casos para actuar indirectamente sobre el proceso inflamatorio, al eliminar los microorganismos residuales en la parte apical del conducto (Gulabivala $\& N G, 2014)$.

\section{Hidróxido de Calcio}

El hidróxido de calcio se utiliza ampliamente como medicamento intraconducto, pues ayuda a eliminar bacterias, reducir la inflamación, eliminar el exudado apical, controlar la reabsorción inflamatoria de la raíz y prevenir la contaminación entre citas, pues actúa como una barrera física que impide el ingreso bacteriano y destruye las bacterias residuales dentro del sistema de conductos radiculares (Mohammadi \& Dummer, 2011). La duración del apósito con hidróxido de calcio, si se utiliza con el objetivo de detener un conducto con exudado persistente, debe ser por un período de al menos 14 días (Gulabivala \& NG, 2014).

El posible mecanismo de acción del hidróxido de calcio en el control del exudado persistente puede deberse a sus propiedades antibacterianas, al ser capaz de dañar la membrana citoplasmática bacteriana y causar desnaturalización de proteínas y daños en el ADN. También se ha propuesto que la liberación de iones hidroxilo y el cambio de pH en el proceso de alcalinización del hidróxido de calcio, proporcionan un ambiente que favorece la reparación y calcificación (Chong \& Pitt Ford, 1992). Además, la reducción del exudado también obedece a las propiedades higroscópicas del hidróxido de calcio (Peters et al., 2005; Fava \& Saunders, 1999).

Otro mecanismo de acción sugerido incluye la vasoconstricción, ya que un estudio (Kikuchi, 2003) investigó si las soluciones de hidróxido de calcio, aplicadas durante 10 segundos, causaban la constricción de microvasos en el lecho de microcirculación del mesenterio de ratas. Se evidenció que en las arteriolas se generaba una vasoconstricción rápida y transitoria, mientras que en los capilares y vénulas, no se produjo una constricción estadísticamente significativa; la constricción arteriolar podría atribuirse a la liberación de calcio. El efecto vasoconstrictor del hidróxido de calcio, resultaría en el control del exudado persistente, ya que la vasoconstricción arteriolar conduce a una reducción en el flujo sanguíneo venular y, bajo condiciones inflamatorias, la reducción de éste último, contribuiría a la disminución de la filtración plasmática, ya que esta se da a través de las paredes venulares (Kikuchi, 2003).

\section{Pasta triple antibiótica}

La pasta triple antibiótica (ciprofloxacina, minociclina y metronidazol) ha demostrado ser útil como medicación intraconducto en los canales con exudado persistente, cuando la infección persiste luego de la terapia con hidróxido de calcio. Así lo demuestra un reporte de caso de un diente \#44 con dens evaginatus (Ayer et al., 2015), en el que a pesar de terapia con hidróxido de calcio durante 2 meses, persistían el exudado intraconducto y el tracto sinusal. En este punto del tratamiento se realizaron un cultivo y test de sensibilidad con varios medicamentos, siendo la pasta triple antibiótica la que mostró la zona de inhibición más grande. Se procedió a cambiar la medicación intraconducto, reemplazando al hidróxido de calcio por la pasta triple antibiótica, haciendo recambios cada 15 días, durante un período de tres meses. Luego de este período, habían ya remitido el exudado y el tracto sinusal, por lo que se procedió a obturar el conducto radicular; a los 6 meses se evidenció reparación de la lesión periapical (Ayer et al., 2015).

\section{Aspiración mediante sistema EndoVac}

En casos de lesiones periapicales grandes, siempre que sea posible, el clínico debería buscar un abordaje no quirúrgico para el manejo de estas lesiones. Un estudio ha evidenciado un método alternativo para el manejo no quirúrgico de una gran lesión 
periapical parecida a quiste en una pieza dental \# 31, mediante descompresión a través del espacio del conducto radicular, empleando el sistema de presión negativo EndoVac, durante 5 citas de tratamiento (Keleş \& Alçin, 2015).

La técnica provoca la disminución de la presión hidrostática dentro de la gran lesión periapical, al resultar en el drenaje de líquido a través del conducto, ya que la presión negativa generada por el EndoVac conduce a la aspiración de este líquido (Keleş \& Alçin, 2015). Por otro lado, esta aspiración es más probable que ocurra en los quistes en bolsa que en los quistes verdaderos y, se ha sugerido que es más probable que, a diferencia de estos últimos, los quistes en bolsa cicatricen después de terapia endodóntica convencional. En contraste la dinámica tisular de un quiste verdadero apical es autosuficiente, porque está completamente separado del sistema de conductos radiculares (Nair et al, 1993). En este reporte de caso se logró la curación de una gran lesión periapical mediante el diagnóstico correcto y el enfoque de tratamiento adecuado, sin necesidad de cirugía periapical, resultando el empleo del sistema de irrigación negativo EndoVac beneficioso para la aspiración intraconducto de exudados, por lo que este dispositivo podría ser útil en el caso de presencia de exudado persistente.

\section{Cirugía endodóntica}

Un reporte de caso (Ferreira, 2004) ilustra el retratamiento endodóntico (luego de dos tratamientos fallidos) de un premolar superior con fístula y lesión periapical, en el que los conductos presentaban constante exudado, por lo que la medicación intraconducto (hidróxido de calcio) fue remplazada mensualmente, hasta que finalmente, luego de un período de 13 meses, se consiguió el secado de los conductos. Sin embargo, luego de la obturación definitiva, la fístula seguía presente y drenaba exudado, por lo que se planificó la cirugía endodóntica. El ápice radicular resecado fue analizado mediante microscopio electrónico de barrido, evidenciándose la presencia de una infección extrarradicular persistente, la cual inherentemente nunca fue afectada por la acción de agentes antimicrobianos como los irrigantes y la medicación intraconducto utilizados durante el tratamiento de conductos.

Este caso pone de manifiesto que luego de un tratamiento endodóntico, que incluye la instrumentación, la irrigación y la colocación de hidróxido de calcio, la invasión bacteriana a veces persiste y en ciertos casos es imposible el control del exudado persistente, siendo entonces una buena alternativa la cirugía periapical, al realizar la resección del extremo apical de la raíz, eliminando así de manera definitiva la infección refractaria establecida, y promoviendo la reparación de los tejidos periapicales (Ferreira, 2004). Así mismo, los reportes de caso previamente citados (Ricucci, 2013; Santos Soares, 2016) en el apartado de causa bacteriana o infección recalcitrante, requirieron finalmente de cirugía apical, ya que durante varias sesiones se presentó exudado persistente en los conductos radiculares, el cual nunca remitió por completo, a pesar del empleo de hidróxido de calcio como medicación intraconducto durante múltiples citas.

\section{Terapia con láser Nd:YAG}

En un estudio (Hassan, 1995) se evaluó tanto clínica como histopatológicamente el efecto del láser Nd:YAG en el manejo de conductos con exudado persistente. Se evidenció que el láser Nd:YAG, aplicado en la posición apical de los conductos, reduce la inflamación apical y acelera el secado de la luz del conducto, empleando como parámetros 15pps (pulsos por segundo) y 1,5 W, durante 2-2,5 minutos. Se obtuvo un secado completo de los conductos en el $82 \%$ de los casos y, la mayoría de los dientes tratados se volvieron asintomáticos. Por otro lado, el examen histopatológico de la lesión periapical relacionada con los conductos con exudado persistente, mostró una marcada mejoría en la condición inflamatoria (Hassan, 1995).

Aunque no se ha establecido de manera precisa el mecanismo por el cual el láser de Nd:YAG ayuda en el control del exudado persistente, ello podría obedecer a la serie de mecanismos biomoleculares involucrados al emplear láser terapéutico o láser 
de bajo nivel, a lo que actualmente se conoce como fotobiomodulación, cuyos mecanismos a nivel molecular y celular están descritos de manera extensa en la literatura (Hamblin, 2017; Glass, 2021; Dompe, 2020; de Freitas \& Hamblin, 2016), y que por asuntos de espacio no pueden abordase en la presente revisión.

La ventaja de la fotobiomodulación es que es una terapia no invasiva que estimula los procesos biológicos naturales y favorece las reacciones de óxido-reducción en las células. Una célula en un estado redox bajo es ácida, pero después de la irradiación con láser, se vuelve más alcalina y puede funcionar de manera óptima. El efecto más esencial puede ser el aumento de trifosfato de adenosina (ATP); la luz láser disociará la unión entre el óxido nítrico (NO) y la citocromo c oxidasa, lo que le permitirá a la célula reanudar la producción de ATP. Este mecanismo básico inicia una cascada de señalización celular, particularmente activación de una serie de factores de transcripción, que conduce a una optimización de las funciones corporales (Convissar, 2015).

Se ha evidenciado que la fotobiomodulación estimula la reparación tisular, alivia el dolor y reduce los niveles de inflamación (Hamblin, 2017; Glass, 2021; Dompe, 2020; de Freitas \& Hamblin, 2016). Se han descrito varios mecanismos anti-inflamatorios relacionados con la aplicación de la fotobiomodulación sobre tejidos inflamados, los cuales podrían fundamentar el empleo de esta terapia en el manejo de los conductos con exudado persistente. Entre estos mecanismos destacan: (Hamblin, 2017; Freitas \& Hamblin, 2016; Convissar, 2015) a) reduce la expresión de factor de necrosis tumoral (TNF), b) reduce los niveles de especies reactivas de oxígeno, c) aumenta la expresión de mecanismos antioxidantes, d) reduce la expresión de especies reactivas de nitrógeno y prostaglandinas, e) reduce los niveles de citocinas proinflamatorias en células inflamatorias activadas, f) reduce los marcadores de fenotipo M1 en macrófagos activados, g) reduce el edema tisular al disminuir la permeabilidad y aumentar el lumen de los vasos linfáticos.

Por otro lado, aunque el empleo de la terapia con láser Nd:YAG parecería promisorio, únicamente se encontró el estudio previamente citado (Hassan, 1995) para el manejo clínico del conducto con exudado persistente, por lo que el empleo de esta técnica requiere inherentemente de más estudios clínicos, que respalden de manera fundamentada su uso en estos casos.

\section{Conclusiones}

El conducto con exudado persistente o conducto llorón, es una entidad clínica que en ocasiones se presenta durante la terapia endodóntica. La causa principalmente descrita es la microbiana, en forma de biofilm bacteriano o infección recalcitrante. Respecto a su manejo clínico, existen disponibles una serie de abordajes, como las puntas de papel, la medicación intraconducto con hidróxido de calcio y sistemas de aspiración como el EndoVac, en tanto que el empleo de la terapia con láser Nd:YAG requiere de más estudios que respalden su uso clínico. Finalmente, en los casos que no se pueda controlar el exudado, a pesar del empleo de uno o más abordajes, y se sospeche de infección recalcitrante, la cirugía endodóntica es la elección.

Por último, es evidente que se requieren más estudios para determinar los agentes causales y el manejo apropiado del conducto con exudado persistente. En este sentido, a futuro se debería incentivar la realización de estudios moleculares de identificación bacteriana, con la finalidad de identificar microorganismos que puedan estar implicados en la génesis y/o mantenimiento del conducto con exudado persistente. Así mismo, en razón del inherente carácter inflamatorio de esta condición clínica, se debería incentivar estudios encaminados a determinar mecanismos moleculares inflamatorios que podrían estar implicados, como podría ser por ejemplo, la carencia o disminución de factores esenciales en la fase de resolución de la inflamación, como la superfamilia de los mediadores pro-resolución especializados (Panigrahy et al., 2021; Serhan et al., 2020), lo que podría derivar incluso en posibles aplicaciones terapéuticas futuras. 
Research, Society and Development, v. 10, n. 9, e52910918558, 2021

(CC BY 4.0) | ISSN 2525-3409 | DOI: http://dx.doi.org/10.33448/rsd-v10i9.18558

\section{Referencias}

Alshahrani, M., DiVito, R., \& DiVito E. (2016). Root Canal Catheterization. En Alshahrani M, DiVito E, De Moor R (Eds.), Lasers in Endodontics. (pp. 40). Cham: Springer International Publishing.

Ayer, A., Vikram, M., \& Suwal, P. (2015). Dens Evaginatus: A Problem-Based Approach. Case reports in dentistry, 2015, 393209. https://doi.org/10.1155/2015/393209

Baumgartner, J. C., Watkins, B. J., Bae, K. S., \& Xia, T. (1999). Association of black-pigmented bacteria with endodontic infections. Journal of endodontics, 25(6), 413-415. https://doi.org/10.1016/S0099-2399(99)80268-4

Bhaskar S. N. (1972). Nonsurgical resolution of radicular cysts. Oral surgery, oral medicine, and oral pathology, 34(3), 458-468. https://doi.org/10.1016/00304220(72) $90325-8$

Brown D. (2017). Paper points revisited: risk of cellulose fibre shedding during canal length confirmation. International endodontic journal, 50(6), 620-626. https://doi.org/10.1111/iej.12663

Chong, B. S., \& Pitt Ford, T. R. (1992). The role of intracanal medication in root canal treatment. International endodontic journal, 25(2), 97-106. https://doi.org/10.1111/j.1365-2591.1992.tb00743.x

Convissar, R. A. (2015) Principles and Practice of Laser Dentistry - E-Book. Elsevier Health Sciences.

de Freitas, L. F., \& Hamblin, M. R. (2016). Proposed Mechanisms of Photobiomodulation or Low-Level Light Therapy. IEEE journal of selected topics in quantum electronics: a publication of the IEEE Lasers and Electro-optics Society, 22(3), 7000417. https://doi.org/10.1109/JSTQE.2016.2561201

Dompe, C., Moncrieff, L., Matys, J., Grzech-Leśniak, K., Kocherova, I., Bryja, A., Bruska, M., Dominiak, M., Mozdziak, P., Skiba, T., Shibli, J. A., Angelova Volponi, A., Kempisty, B., \& Dyszkiewicz-Konwińska, M. (2020). Photobiomodulation-Underlying Mechanism and Clinical Applications. Journal of clinical medicine, 9(6), 1724. https://doi.org/10.3390/jcm9061724

Durack C., \& Brady E. (2019). Root Canal Filling. En Patel S, Barnes J (Eds.), The principles of Endodontics. (3ra ed., pp.103) NewYork, NY: Oxford University Press.

Estrela C, (2018). Metodologia Científica: Ciência, Ensino, Pesquisa. Editora Artes Médicas.

Fava, L. R., \& Saunders, W. P. (1999). Calcium hydroxide pastes: classification and clinical indications. International endodontic journal, 32(4), $257-282$. https://doi.org/10.1046/j.1365-2591.1999.00232.x

Ferreira, F. B., Ferreira, A. L., Gomes, B. P., \& Souza-Filho, F. J. (2004). Resolution of persistent periapical infection by endodontic surgery. International endodontic journal, 37(1), 61-69. https://doi.org/10.1111/j.1365-2591.2004.00753.x

Glass G. E. (2021). Photobiomodulation: A review of the molecular evidence for low level light therapy. Journal of plastic, reconstructive \& aesthetic surgery: JPRAS, 74(5), 1050-1060. https://doi.org/10.1016/j.bjps.2020.12.059

Gomes, B. P., Drucker, D. B., \& Lilley, J. D. (1994). Associations of specific bacteria with some endodontic signs and symptoms. International endodontic journal, 27(6), 291-298. https://doi.org/10.1111/j.1365-2591.1994.tb00271.x

Gomes, B. P., Pinheiro, E. T., Gadê-Neto, C. R., Sousa, E. L., Ferraz, C. C., Zaia, A. A., Teixeira, F. B., \& Souza-Filho, F. J. (2004). Microbiological examination of infected dental root canals. Oral microbiology and immunology, 19(2), 71-76. https://doi.org/10.1046/j.0902-0055.2003.00116.x

Gulabivala, K., \& Ng, Y-L. (2014). Management of non-surgical root-canal treatment failure. En Gulabivala K, Ng, Y-L (Eds.), Endodontics. (4a ed.) China: Mosby.

Gutmann, J., \& Lovdahl, P. (2014). Problem-Solving Clinical Techniques in Enlarging and Shaping the Root Canal. En Gutmann J, Lovdahl P (Eds.), Problem Solving in Endodontics Prevention. (5a ed.).China: Mosby.

Hamblin M. R. (2017). Mechanisms and applications of the anti-inflammatory effects of photobiomodulation. AIMS biophysics, 4(3), 337-361. https://doi.org/10.3934/biophy.2017.3.337

Hassan F. E. (1995). A new method for treating weeping canals: clinical and histopathologic study. Egyptian dental journal, 41(4), 1403-1408.

Kahler, B. (2015). Healing of a cyst-like lesion involving an implant with nonsurgical management. Journal of endodontics, 41(5), 749-752. https://doi.org/10.1016/j.joen.2014.12.013

Keleş, A., \& Alçin, H. (2015). Use of EndoVac System for Aspiration of Exudates from a Large Periapical Lesion: A Case Report. Journal of endodontics, 41(10), 1735-1737. https://doi.org/10.1016/j.joen.2015.05.019

Kikuchi, I., Wadachi, R., Yoshioka, T., Okiji, T., Kobayashi, C., \& Suda, H. (2003). An experimental study on the vasoconstriction effect of calcium hydroxide using rat mesentery. Australian endodontic journal: the journal of the Australian Society of Endodontology Inc,29(3), 116-119. https://doi.org/10.1111/j.17474477.2003.tb00532.x 
Research, Society and Development, v. 10, n. 9, e52910918558, 2021

(CC BY 4.0) | ISSN 2525-3409 | DOI: http://dx.doi.org/10.33448/rsd-v10i9.18558

Koche, J. C. (2011). Fundamentos de metodologia científica. Vozes. http://www.brunovivas.com/wp-content/uploads/sites/10/2018/07/K\%C3\%B6che-Jos\%C3\%A9Carlos0D0AFundamentos-de-metodologia-cient\%C3\%ADfica-_teoria-da0D0Aci\%C3\%AAncia-e-inicia\%C3\%A7\%C3\%A3o-\%C3\%A0pesquisa.pdfhttps://repositorio.ufsm.br/bitstream/handle/1/15824/Lic_Computacao_Metodologia-Pesquisa-Cientifica.pdf?sequence=1

Koppang, H. S., Koppang, R., Solheim, T., Aarnes, H., \& Stølen, S. O. (1989). Cellulose fibers from endodontic paper points as an etiological factor in postendodontic periapical granulomas and cysts. Journal of endodontics, 15(8), 369-372. https://doi.org/10.1016/S0099-2399(89)80075-5

Lomçali, G., Sen, B. H., \& Cankaya, H. (1996). Scanning electron microscopic observations of apical root surfaces of teeth with apical periodontitis. Endodontics \& dental traumatology, 12(2), 70-76. https://doi.org/10.1111/j.1600-9657.1996.tb00100.x

Mohammadi, Z., \& Dummer, P. M. (2011). Properties and applications of calcium hydroxide in endodontics and dental traumatology. International endodontic journal, 44(8), 697-730. https://doi.org/10.1111/j.1365-2591.2011.01886.x

Nair, P. N., Sjögren, U., Schumacher, E., \& Sundqvist, G. (1993). Radicular cyst affecting a root-filled human tooth: a long-term post-treatment followup. International endodontic journal, 26(4), 225-233. https://doi.org/10.1111/j.1365-2591.1993.tb00563.x

Natkin, E., Oswald, R. J., \& Carnes, L. I. (1984). The relationship of lesion size to diagnosis, incidence, and treatment of periapical cysts and granulomas. Oral surgery, oral medicine, and oral pathology, 57(1), 82-94. https://doi.org/10.1016/0030-4220(84)90267-6

Panigrahy, D., Gilligan, M. M., Serhan, C. N., \& Kashfi, K. (2021). Resolution of inflammation: An organizing principle in biology and medicine. Pharmacology \& therapeutics, 107879. Advance online publication. https://doi.org/10.1016/j.pharmthera.2021.107879

Pereira, A. S., Shitsuka, D. M., Parreira, F. J., \& Shitsuka, R. (2018). Metodología de la investigación científica.[libro electrónico]. Santa María. Ed. UAB/NTE/UFSM. Disponible en: https://repositorio. ufsm. br/bitstream/handle/1/15824/Lic_Computacao_Metodologia-Pesquisa-Cientifica. pdf.

Peters, C. I., Koka, R. S., Highsmith, S., \& Peters, O. A. (2005). Calcium hydroxide dressings using different preparation and application modes: density and dissolution by simulated tissue pressure. International endodontic journal, 38(12), 889-895. https://doi.org/10.1111/j.1365-2591.2005.01035.x

Ricucci, D., Candeiro, G. T., Bugea, C., \& Siqueira, J. F., Jr (2016). Complex Apical Intraradicular Infection and Extraradicular Mineralized Biofilms as the Cause of Wet Canals and Treatment Failure: Report of 2 Cases. Journal of endodontics, 42(3), 509-515. https://doi.org/10.1016/j.joen.2015.12.014

Santos Soares, S. M., Brito-Júnior, M., de Souza, F. K., Zastrow, E. V., Cunha, C. O., Silveira, F. F., Nunes, E., César, C. A., Glória, J. C., \& Soares, J. A. (2016). Management of Cyst-like Periapical Lesions by Orthograde Decompression and Long-term Calcium Hydroxide/Chlorhexidine Intracanal Dressing: A Case Series. Journal of endodontics, 42(7), 1135-1141. https://doi.org/10.1016/j.joen.2016.04.021

Serhan, C. N., Gupta, S. K., Perretti, M., Godson, C., Brennan, E., Li, Y., Soehnlein, O., Shimizu, T., Werz, O., Chiurchiù, V., Azzi, A., Dubourdeau, M., Gupta, S. S., Schopohl, P., Hoch, M., Gjorgevikj, D., Khan, F. M., Brauer, D., Tripathi, A., Cesnulevicius, K., \& Wolkenhauer, O. (2020). The Atlas of Inflammation Resolution (AIR). Molecular aspects of medicine, 74, 100894. https://doi.org/10.1016/j.mam.2020.100894

Signoretti, F. G., Gomes, B. P., Montagner, F., \& Jacinto, R. C. (2013). Investigation of cultivable bacteria isolated from longstanding retreatment-resistant lesions of teeth with apical periodontitis. Journal of endodontics, 39(10), 1240-1244. https://doi.org/10.1016/j.joen.2013.06.018

Siqueira J. F., Jr (2001). Aetiology of root canal treatment failure: why well-treated teeth can fail. International endodontic journal, 34(1), 1-10. https://doi.org/10.1046/j.1365-2591.2001.00396.x

Siqueira, J. F., Rôças, I. N., \& Lopes, H. (2015) Medicação Intracanal. En Siqueira JF, Lopes H (Eds.), Endodontia: Biologia e Técnica. (4a ed.). Brasil:Elsevier.

Siqueira, J. F., \& Rôças, I. N. (2016) Intracanal Medication. En Chong BS (Ed.) Harty’s Endodontics in Clinical Practice. (7ma ed., pp.141). Churchill Livingstone.

Siqueira, J. F., \& Rôças, I. N. (2020) Microbiology of Apical Periodontitis. En Ørstavik D, (Ed.), Essential endodontology: Prevention and Treatment of Apical Periodontitis. (3ra ed., pp 121) Hoboken, NJ: Wiley-Blackwell.

Siqueira, J. F., Jr, \& Rôças, I. N. (2013). Microbiology and treatment of acute apical abscesses. Clinical microbiology reviews, 26(2), 255-273. https://doi.org/10.1128/CMR.00082-12

Sousa, E. L., Martinho, F. C., Nascimento, G. G., Leite, F. R., \& Gomes, B. P. (2014). Quantification of endotoxins in infected root canals and acute apical abscess exudates: monitoring the effectiveness of root canal procedures in the reduction of endotoxins. Journal of endodontics, 40(2), 177-181. https://doi.org/10.1016/j.joen.2013.10.008

Sundqvist, G., Johansson, E., \& Sjögren, U. (1989). Prevalence of black-pigmented bacteroides species in root canal infections. Journal of endodontics, 15(1), 1319. https://doi.org/10.1016/S0099-2399(89)80092-5

Tronstad, L. (2003). Endodontic Complications. En Tronstad L (Ed.), Clinical Endodontics. (2a ed.), Thieme.

Walton, R. E., \& Keiser, K. (2009). Endodontic Emergencies and Therapeutics. En Torabinejad M, Walton RE (Eds.), Endodontics: Principles and Practice. (4ta ed., pp 156). St. Louis, Mo: Saunders/Elsevier.

Weine, F. S.. (2004) Endodontic therapy. Mosby.

Yoshino, A., Tabuchi, M., Uo, M., Tatsumi, H., Hideshima, K., Kondo, S., \& Sekine, J. (2013). Applicability of bacterial cellulose as an alternative to paper points in endodontic treatment. Acta biomaterialia, 9(4), 6116-6122. https://doi.org/10.1016/j.actbio.2012.12.022

Zhang, C., Du, J., \& Peng, Z. (2015). Correlation between Enterococcus faecalis and Persistent Intraradicular Infection Compared with Primary Intraradicular Infection: A Systematic Review. Journal of endodontics, 41(8), 1207-1213. https://doi.org/10.1016/j.joen.2015.04.008 